\title{
Radionuclide Bone Scintigraphy: An Interesting Case Report
}

\author{
${ }^{1}$ Mohana Hossain, ${ }^{2}$ Hosne Ara Rahman, ${ }^{2}$ Mahbubul Haque, ${ }^{2}$ Mahbubur Rahman, ${ }^{1}$ Md. Sanowar \\ Hossain, ${ }^{1}$ Fatema Sultana Haque, ${ }^{1}$ Rubina Begum, ${ }^{1}$ Shankar Kumar Biswas, ${ }^{2}$ Md. A.B. Siddique \\ ${ }^{1}$ Institute of Nuclear Medicine and Allied Sciences, Dhaka Medical College campus, Dhaka, BAEC \\ ${ }^{2}$ Institute of Nuclear Medicine and Allied Sciences, Sir Salimullah Medical College campus, Mitford, \\ BAEC
}

Correspondence: Mohana Hossain, Medical Officer, INMAS, Dhaka

Email: mohana_hossain@yahoo.com

\begin{abstract}
It is well established that Technetium ${ }^{99 m}$ methylene diphosphonate (Tc ${ }^{99 m}$ MDP) whole body bone scintigraphy (WBBS) can demonstrate multiple lesions with increased radiotracer concentration in involved bone. But it is hard to differentiate multiple benign osteolytic lesions from disseminated bone metastases. Even combined with medical history and multiple imaging results, clinical diagnosis of metastatic lesion remains a challenge. This can affect the treatment procedure. Here the role of skeletal scintigraphy in a case of eosinophilic granuloma is evaluated and concluded that additional attention should be given before diagnosing any case as bone metastases.
\end{abstract}

\section{INTRODUCTION}

Eosinophilic granuloma (EG) of bones is the commonest benign manifestation of Histiocytosis$\mathrm{X}$ malady (1). There is a certain predilection to males (2:1) (2). Usually it is asymptomatic but may appear as a palpable, tender mass over the affected area. Tc ${ }^{99 m}$ MDP WBBS is commonly used to detect metastases from malignant tumor as it is more sensitive than X-rays and allow the whole body to be surveyed (3). In bone scintigraphy eosinophilic granuloma, multiple myeloma, disseminated tuberculosis, enchondroma and several other diseases show multiple photopenic lesions that is similar with the findings of malignant bone metastases. Therefore, prior to delivering the report of metastases, benign osteolytic lesions should be considered according to other imaging findings, detailed medical history, or even the preliminary pathology result. A case is reported here where the patient developed EG involving multiple bones. 


\section{CASE REPORT}

A 30-year-old male was referred to CNMU, Mitford on $5^{\text {th }}$ July 2013 to have a skeletal scintigraphy. He mentioned a palpable, soft, immobile mass on the right side of skull. He also mentioned loosening of lower molar and premolar teeth. No family history of bony tumor was recorded. The physical and neurological examination had no pathological signs. Blood examination was unremarkable except raised ESR. Plain radiographs demonstrated a large (2.1 $\mathrm{cm}$ ) oval-shaped osteolytic area in right parietal bone along with two more similar lesions in left parietal and temporal bone. Lesions were also visible in mandible. MRI of skull and thoracic CT scan also indicated lesions favoring bone metastases. Tc ${ }^{99 m}$ MDP WBBS was performed and in addition to skull, it revealed increased radiotracer uptake in different areas of bone (vertebrae, ribs, lower end of femora and tibiae) mimicking bone metastases as other radiographic findings. FNAC of the skull mass showed many Langerhans cells, eosinophils and lymphocytes which set the diagnosis of EG of skull. A radical excision of the largest skeletal lesion was performed followed by 3 cycles of chemotherapy. Follow up skull CT after 6 months showed no further lesion on the operated site. A follow up bone scan was also performed and similar findings (except for the largest skull lesion) as previous scan were observed.

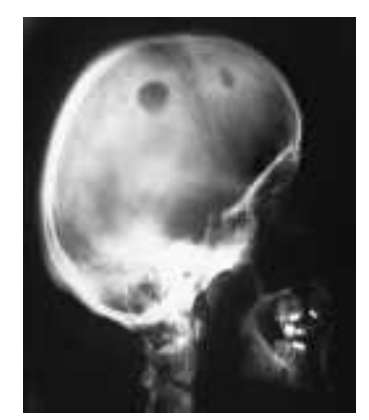

Figure 1: CT scan of skull demonstrating multiple osteolytic lesions.

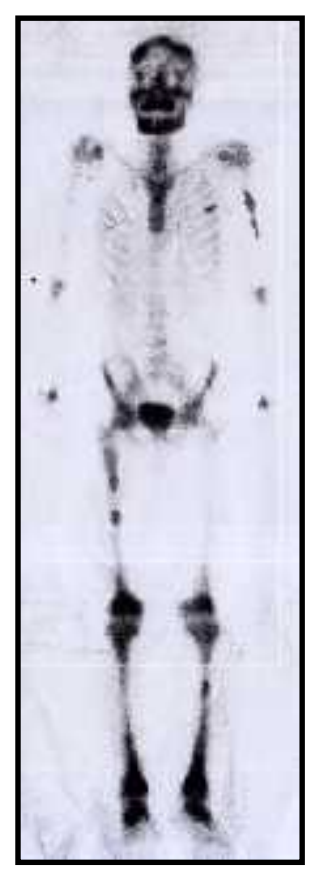

Figure 2: Whole body Bone scan demonstrating increased radiotracer uptake in several areas of bone. 


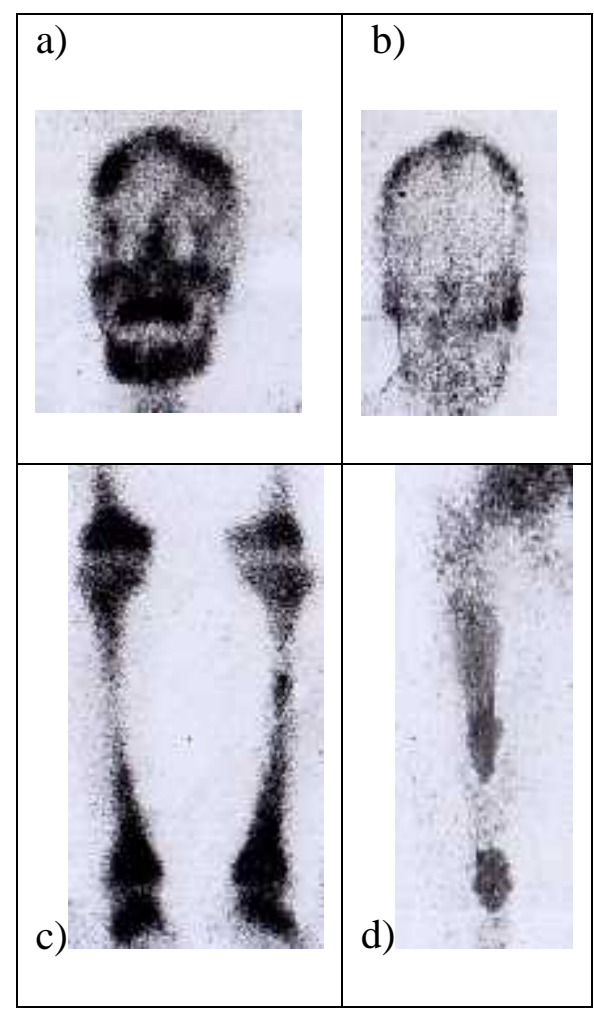

Figure 3: Magnified images of increased uptake in several areas of skull and mandible (a \& b); lower end of femora, proximal and distal end of tibiae, left tibial shaft (c); shaft of right femur (d)

\section{DISCUSSION}

Eosinophilic granuloma (EG) is one of the rarest bone tumor ( $<1 \%$ of bone tumor) affecting mostly the children (90\%) and adolescent (3). Other forms of EG are Hand-Schüller-Christian disease (A triad of Diabetes Insipidus, exophthalmos and lytic bone lesions) and the Letterer-Siwe Syndrom (A rapidly progressing multisystem disease with poor prognosis). Solitary lesion is more common than multiple lesions (4). But in this case, a 30-year-old male with EG was referred to this centre for Tc ${ }^{99 \mathrm{~m}}$ MDP bone scan having multiple metastases like bone involvement on radiographic imaging. Even though the patient had multiple osteolytic lesions, it can be easily distinguished from Hand-Schüller Christian disease for the absence of characteristic Diabetes Insipidus and exophthalmos.

EG can be asymptomatic or presented as local swelling, pain, tenderness, neurological symptoms, fracture, loosening of teeth, otitis media, exophthalmos or hemangioma (5). Visceral involvement is rare. 
The patient in this case presented with a soft immobile swelling on skull with loosening of teeth in lower jaw.

Etiology of EG remains unknown but recently there have been some assumptions like inflammatory processes, autoimmune disorders and an out of control proliferation of Langerhans cell (6). It is now known that this exerts Interleukin-1 and Prostaglandin $\mathrm{E}_{2}$ leading to damage to the surrounding tissue. However in this case exact etiology could not be identified.

Radiographic skeletal survey is the main modality for the diagnosis of EG, even though the radiographic characteristics of the disease considerably vary according to the site and the activity of the lesion. Bone destruction in granulation tissue forming areas of osteolysis is the main pathological characteristic of EG and may involve any portion of bone. In this case plain radiograph, CT and MRI of skull depicted multiple extensions describing their size, borders, situation of surrounding tissues with the tumor extension. Tc ${ }^{99 m}$ MDP WBBS was less sensitive in depicting these details of the osseous lesion. But bone scintigraphy played a vital role in figuring out the additional bone lesions (in vertebra, rib, femora and tibiae in addition to skull and mandible lesion) in single setting and monitoring therapeutic response. According to some authors it is also helpful when the radiographs are normal or equivocal $(4,6)$.

In addition, $\mathrm{Tc}^{99 \mathrm{~m}}$ MDP WBBS has the advantage of detecting asymptomatic bone abnormalities possibly missed by other imaging modalities. Combination of bone scintigraphy and radiographic survey might be helpful to make a definite diagnosis when bone biopsy or operation is not possible. Howarth et al.1996 in a survey found excellent role of skeletal scintigraphy in diagnosis and staging of LCH [91\% sensitive] (7). In this patient all images of plain radiographs, CT, MRI and $\mathrm{Tc}^{99 \mathrm{~m}}$ MDP demonstrated the characteristics of disseminated bone metastasis although EG was finally diagnosed by histopathology report. Radiation dosimetry to adult reproductive organs was less favorable for radiographic skeletal survey compared to bone scintigraphy and strongly supports its use in subsequent follow up (7)

\section{CONCLUSION:}

Multiple benign osteolytic lesions may lead to a false positive diagnosis for metastatic bone lesions. So, care should be taken before giving a final diagnosis of disseminated bone metastasis. Detailed past medical history, various laboratory, radiographic and pathologic results should also be accounted to lead to a correct diagnosis. Moreover, less radiation hazard comparing to other radiologic modalities has made skeletal scintigraphy a more sensitive tool in monitoring the patient's progress and allows the facility of whole body survey in a single setting. 


\section{REFERENCE}

1. Chada M, Agarwal A, Agarwal N, Singh MK. Solitary eosinophilic granuloma of the radius. An unusual differential diagnosis.ActaOrthopBelg 2007, 73:413-417.

2. Ando A, Hatori M, Hosaka M, Hagiwara Y, Kita A, Itoi E. Eosinophilic granuloma arising from the pelvis in children: a report of three cases.Upsala J Med Sci 2008, 113(1): 209-216.

3. Solav S. Bone Scintiscanning in Osteolytic Lesions. Clinical Nuclear Medicine 2004, 29(1):1220.

4. Monroc M, Pointe HD, Haddad S, Josset P, Montage JP. Soft tissue signal abnormality associated with eosinophilic granuloma: correlation of MR imaging with pathologic findings.PediatrRadiol 1994, 24:328-332.

5. Donadieu J. A multicentre retrospective survey of Langerhans' cell histiocytosis. 348 cases observed between 1983 and 1993. Archives of Disease in Childhood.1996; 75(1):17-24.

6. Kitsoulis PV, Paraskevas G, Vrettakos A and Marini A.A case of eosinophilic granuloma of the skull in an adult man: a case report. Cases Journal 2009, 2:9144.doi: 10.1186/1757-1626-2-9144.

7. Howarth DM, Mullan BP, Wiseman GA, Wenger DE, Forstrom LA, Dunn WL. Langerhans cell histiocytosis (eosinophilic granuloma) of bone in children. J Nucl Med. 1996; 37 (9): 1456. 